Orthopäde $2021 \cdot 50: 528-537$

https://doi.org/10.1007/s00132-021-04121-9

Angenommen: 5. Mai 2021

Online publiziert: 22. Juni 2021 2022

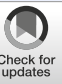

(c) Der/die Autor(en) 2022, korrigierte Publikation

\section{Dynamische Analyse der Gelenkbelastung bei Beinachsendeformitäten in der Frontalebene}

\author{
Stellenwert der instrumentellen Ganganalyse
}

\author{
Felix Stief' · Jana Holder ${ }^{1} \cdot$ Harald Böhm ${ }^{2,3}$ - Andrea Meurer \\ 'Klinik für Orthopädie (Friedrichsheim), Universitätsklinikum Frankfurt, Frankfurt am Main, Deutschland \\ ${ }^{2}$ Orthopädische Kinderklinik, Aschau im Chiemgau, Deutschland \\ ${ }^{3}$ PFH Private Hochschule, Göttingen, Deutschland
}

Zusammenfassung

\section{In diesem Beitrag}

- Messmethoden

Kinematische Bewegungsanalyse • Kinetik - Berechnung von Gelenkmomenten • Kinetik - Berechnung von Kontaktkräften im Gelenk mithilfe eines erweiterten muskuloskelettalen Modells • Messung der Muskelaktivität mittels Elektromyographie

- Stellenwert der IGA bei der Beurteilung von Achsfehlstellungen

Die dynamische Gelenkbelastung als Prädiktor für Arthroseentwicklung • Diskrepanz zwischen statischer Achsfehlstellung und dynamischer Gelenkbelastung • Praxisbeispiel • Optimierung des Behandlungsschemas bei der Beurteilung von Achsfehlstellungen durch Integration der IGA

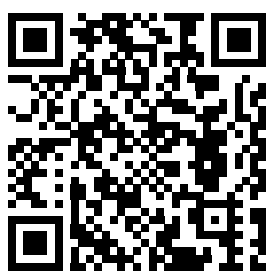

QR-Code scannen \& Beitrag online lesen

Hintergrund: Die instrumentelle 3-D-Ganganalyse (IGA) hat sich zur funktionellen Beurteilung orthopädischer Krankheitsbilder etabliert. Sie kann wertvolle Zusatzinformationen zur konventionellen statischen Röntgendiagnostik liefern und trägt so zur Behandlungsentscheidung und dem Operationserfolg bei. Zur Beurteilung von Achsfehlstellungen kommt ihr bisher nur in wenigen spezialisierten Zentren Bedeutung zu.

Anwendung: Der vorliegende Beitrag stellt die Messmethoden der IGA dar und zeigt deren Nutzen bei der Behandlung von Achsfehlstellungen des Knies in der Frontalebene. Insbesondere die Berechnung der dynamischen Gelenkbelastungen liefert wichtige Erkenntnisse im Hinblick auf die Entwicklung degenerativer Gelenkveränderungen im Knie und ergänzt somit die statische Beurteilung der Beinachse. Es wird ein neuer Behandlungsalgorithmus für die kniegelenknahe Wachstumslenkung im Kindesalter mittels temporärer Hemiepiphysiodese vorgestellt. Die IGA kann dabei insbesondere bei Grenzfällen für die klinische Entscheidungsfindung sehr nützlich sein. Bei einer Diskrepanz zwischen statischer Beinachse und dynamischer Kniegelenkbelastung kann die IGA potenzielle Kompensationsmechanismen beim Gehen aufdecken.

\section{Schlüsselwörter}

Achsfehlstellung $\cdot$ Klinische Entscheidungsfindung $\cdot$ Genu valgum $\cdot$ Genu varum · Knie
Die instrumentelle 3-D-Ganganalyse hat sich als Untersuchungsmethodik zur funktionellen Beurteilung orthopädischer Krankheitsbilder etabliert. Über die Berechnung der dynamischen Gelenkbelastungen liefert sie gerade bei der Beurteilung von Achsfehlstellungen wichtige Erkenntnisse und ergänzt somit die statische Beurteilung der Beinachse. Der vorliegende Beitrag gibt einen Überblick über die Messmethoden der instrumentellen Ganganalyse und zeigt deren Nutzen bei der Behandlung von Achsfehlstellungen.

\section{Einleitung}

Die menschliche Fortbewegung wird durch ein komplexes Zusammenspiel der Knochen, Gelenke, Muskeln, Sehnen und Bänder des Bewegungsapparates ermöglicht. Für den Beobachter des menschlichen Ganges kommen in kurzer Zeit zu viele Informationen zusammen, als dass er alle gleichzeitig erkennen und korrekt verarbeiten könnte. Mithilfe der instrumentellen Ganganalyse (IGA) ist es möglich, Abweichungen vom normalen Gangbild festzustellen und die Ursachen dieser Abweichungen herauszufinden. 
Die IGA wird in der Orthopädie und Unfallchirurgie eingesetzt, um einerseits das funktionelle Resultat verschiedener Behandlungen zu quantifizieren oder andererseits, um das individuelle Bewegungsverhalten von Patienteninnen und Patienten zu evaluieren. Somit hilft die IGA, komplexe Gangbildpathologien, Fehlbelastungen und Kompensationsmuster zu beurteilen. Der IGA wird dabei ein besonders hoher Stellenwert im Rahmen der erweiterten Diagnostik und differenzierten Therapieplanung beigemessen.

\section{Messmethoden}

Die Methode der IGA ist ein in Deutschland offiziell anerkanntes Verfahren zur Funktionsdiagnostik und sowohl in dem Katalog zum Verschlüsseln von Operationen und Prozeduren im stationären Bereich (OPS), als auch in der Gebührenordnung für Ärzte (GOÄ) erfasst. Unterschiedlichste technische Verfahren dienen als Grundlage, die Bewegungen einzelner Körpersegmente (Kinematik) in drei Dimensionen zu dokumentieren und mit Informationen der wirkenden Kraft vom Boden (Kinetik) und der Aktivität der Muskulatur (Elektromyographie) zu ergänzen. Neben einfachen Kamerasystemen zur visuellen kinematischen Analyse finden markerbasierte Systeme, markerlose Systeme sowie videorasterstereografische Systeme im klinischen Umfeld Anwendung. Eine Übersicht über die verschiedenen Messsysteme und deren klinische Anwendung findet sich z.B. in dem Übersichtartikel von Oppelt und Kollegen [17]. Die nachfolgenden Ausführungen beziehen sich exemplarisch auf die am häufigsten verwendete Messmethodik mittels markerbasierter Kamerasysteme.

\section{Abkürzungen}

$\begin{array}{ll}\text { GOÄ } & \begin{array}{l}\text { Gebührenordnung für Ärzte } \\ \text { IGA }\end{array} \\ \text { Instrumentelle Ganganalyse } \\ \text { mLDTA } & \begin{array}{l}\text { "Mechanical lateral distal femoral } \\ \text { angle" }\end{array} \\ & \begin{array}{l}\text { "Mechanical lateral distal tibial } \\ \text { angle" }\end{array} \\ \text { mLPFA } & \begin{array}{l}\text { "Mechanical lateral proximal femoral } \\ \text { angle" }\end{array} \\ \text { mMPTA } & \begin{array}{l}\text { "Mechanical medial proximal tibial } \\ \text { angle" } \\ \text { Operationen- und Prozeduren- } \\ \text { schlüssel }\end{array} \\ \text { OPS } & \end{array}$

\section{Kinematische Bewegungsanalyse}

Für die Erfassung der Bewegung der einzelnen Körpersegmente werden bei der IGA in der Regel lichtreflektierende Kugeln (Marker) mit mehreren Infrarotkameras erfasst (-Abb. 1). Das Kamerasystem sendet Lichtimpulse im Infrarotbereich (unsichtbar für das menschliche Auge) aus, welche von den speziell beschichteten Markern reflektiert werden. Die Kameras erfassen diese Reflexionen und übermitteln sie an den Computer, der daraus die 3-D-Raumkoordinaten aller Marker berechnet.

Anhand der Markerpositionen an definierten anatomischen Stellen (z. B. laterale Femurkondyle) wird ein segmentales anatomisches Koordinatensystem berechnet und eine Beziehung zu einem an der Lage des Knochens orientierten Referenzrahmen hergestellt. Dies erlaubt die Berechnung von Gelenkwinkeln (z. B. Flexion/ Extension im Kniegelenk) und Gelenkzentren während der Bewegung. Die Gelenkwinkelverläufe werden dann in drei Dimensionen (sagittal, frontal und transversal) als Relativbewegung der Segmentkoordinatensysteme zueinander oder zum Raumkoordinatensystem (z. B. Stellung des Beckens) beschrieben. Je nach klinischer Fragestellung kommen verschiedene Computermodelle und "Markersets" zur Anwendung [23].

Die graphische Aufbereitung der Gelenkwinkelverläufe wird dann anhand von Winkel-Zeit-Diagrammen und bezogen auf einen kompletten Gangzyklus dargestellt (-Abb. 2). Der Gangzyklus besteht aus der Abfolge zweier Schritte, in der jedes Bein einmal Stand- und einmal Schwungbein ist. Der Bodenkontakt zu Beginn des Gangzyklus ist der Moment, der als Anfang und damit als 0-\%-Punkt des Gangzyklus bezeichnet wird. Der Moment des nächsten Bodenkontakts desselben Fußes (Referenzbein) markiert das Ende des Gangzyklus (100-\%-Punkt). Gleichzeitig vollzieht das andere Bein (kontralaterales Bein) die gleichen Bewegungsabläufe wie das Referenzbein, allerdings um den Ablauf eines halben Gangzyklus verschoben. Jeder Gangzyklus wird in eine Standphase und eine Schwungphase unterteilt. Die Standphase ist die Periode des Gangzyklus, in der der Fuß auf dem Boden ist. Beim normalen Gehen nimmt sie ca. $60 \%$ des Gangzyklus
Hier steht eine Anzeige. $\underline{n}$ Springer 


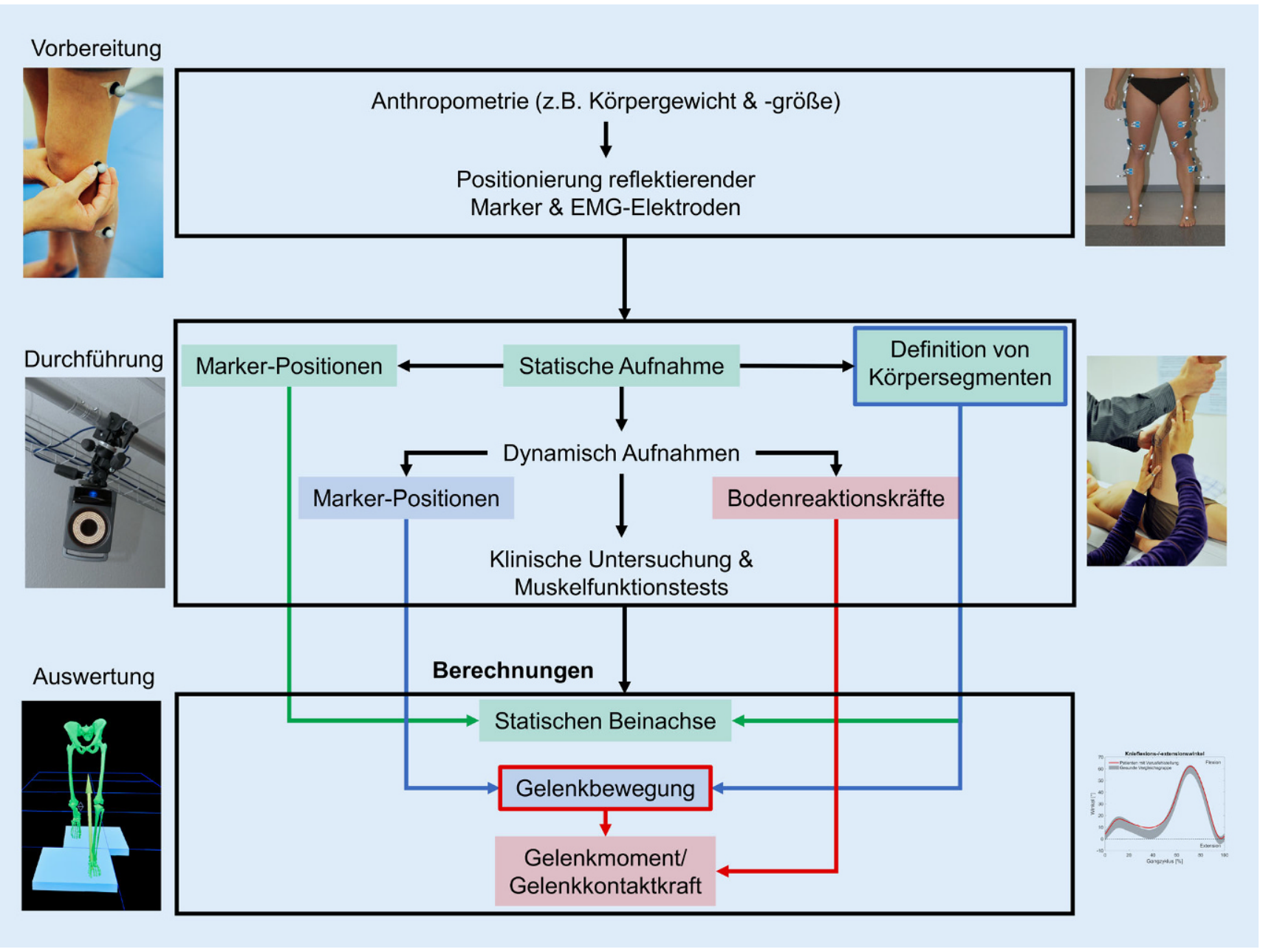

Abb. $1 \Delta$ Schematische Darstellung des Ablaufs einer instrumentellen Ganganalyse

ein. Die Schwungphase bezeichnet den Zeitraum, in dem der Fuß in der Luft ist und unter anderem der Schwung das Bein vorwärtsbringt. Sie beginnt mit dem Abheben des Fußes vom Boden und nimmt beim normalen Gang ca. $40 \%$ des Gangzyklus ein [18]. Durch Übereinanderlegen der Kurven für das rechte und linke Bein lassen sich Symmetrievergleiche durchführen. Mit dem Datenvergleich gesunder Vergleichsgruppen (Normdaten) kann analysiert werden, ob pathologische $\mathrm{Zu}$ stände vorliegen und Behandlungsmaßnahmen eingeleitet werden müssen. $\mathrm{Zu}$ sätzlich lassen sich durch den Vorher-nachher-Vergleich konservative oder operative Therapien evaluieren.

Die Ganganalyse wird von den Probanden in der Regel barfuß, je nach Fragestellung aber auch mit Schuhwerk durchgeführt (z. B. wenn der Effekt einer spezi- ellen orthopädischen Einlage untersucht werden soll).

\section{Kinetik - Berechnung von Gelenkmomenten}

Bodenreaktionskräfte können durch visuelle Beobachtungen nicht erfasst werden. Mit deren Hilfe kann aber auf die Belastung, die auf den Körper und die einzelnen Gelenke einwirkt, geschlossen werden. ZurErfassung der Bodenreaktionskräfte werden im Boden eingelassene Kraftmessplatten verwendet. Durch eine Kombination aus anthropometrischen Informationen, kinematischen Parametern und Bodenreaktionskräften ist es dann über die Berechnungsmethode der sogenannten inversen Dynamik möglich, auf das Gelenk wirkende Momente zu bestimmen, welche sich als indirektes Maß der Gelenkbelastung etabliert haben (- Abb. 3). Dabei werden
Momente um jedes Gelenk über den Abstand des Kraftvektors zum Gelenkzentrum berechnet. Die Gelenkmomente werden dabei in ähnlich standardisierter Form dargestellt wie die Kinematik, d. h. als Kurvendiagramme im Vergleich zu einem Normkollektiv in allen drei Ebenen.

Bezogen auf das Knie sind die Gelenkmomente in der Frontalebene (externes Adduktionsmoment bzw. Varusmoment) von größter Bedeutung hinsichtlich der Knorpeldegeneration/Entstehung von Arthrose im Kniegelenk [2, 9, 13-15, 22]. Bei einer Achsfehlstellung (Genu varum oder Genu valgum) kommt es zu einer Lastverschiebung der Kontaktkraft im Kniegelenk in Bereiche, die auf Dauer nicht für solche Belastungen konditioniert sind [1]. 


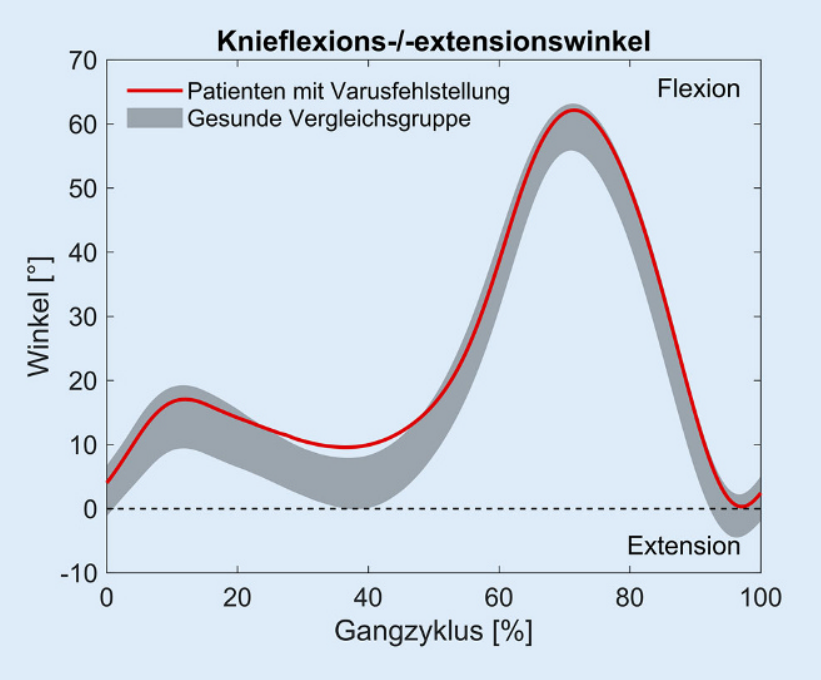

Abb. $2 \Delta$ Exemplarische graphische Aufbereitung der Kniegelenkbewegung in der Sagittaleben anhand eines Winkel-Zeit-Diagramms. Die y-Achse stellt das Bewegungsausmaß im Kniegelenk dar. Die $x$-Achse den Zeitpunkt bezogen auf einen kompletten Gangzyklus. Die rote Kurve stellt eine Patientengruppe mit Varusfehlstellung dar. Im Vergleich zur grau dargestellten gesunden Kontrollgruppe (Mittelwert und Standardabweichung) zeigt sich, bei sonst physiologischem Verlauf der Kniegelenkbewegung, eine eingeschränkte Knieextension in der zweiten Hälfte der Standphase

\section{Kinetik - Berechnung von \\ Kontaktkräften im Gelenk mithilfe eines erweiterten muskuloskelettalen Modells}

Gelenkkontaktkräfte können ein genauerer Indikator für die Gelenkbelastung sein. Erweiterte muskuloskelettale Modelle berücksichtigen bei der Berechnung der Gelenkkontaktkraft neben den externen Kräften auch die Gelenk- und Muskelkräfte (- Abb. 3). Diese Modelle sind in der Lage, körperinterne Strukturen durch die Modellierung von Muskeln, Sehnen, Bänder und Knochen abzubilden. Die bekanntesten Beispiele solcher Systeme zur Erstellung und Simulation biomechanischer Modelle des muskuloskelettalen Systems sind OpenSim (National Center for Simulation in Rehabilitation Research, Stanford, CA, USA) und AnyBody ${ }^{\mathrm{TM}}$ (AnyBody Technology A/S, Aalborg, Dänemark). Eine kürzlich veröffentlichte systematische Literaturübersicht [8] vergleicht die konventionell berechneten resultierenden Gelenkmomente mit den mittels muskuloskelettaler Modellierung berechneten Gelenkkontaktkräften. Es wurde also der Frage nachgegangen, inwiefern die erweiterten muskuloskelettalen Modelle neue bzw. erweiterte Kenntnisse bezüglich der dynamischen Gelenkbelastungen bieten und inwieweit dies kli- nische Schlussfolgerungen beeinflusst. Die Ergebnisse dieser Literaturübersicht zeigen, dass die Wahl der Berechnungsmethode anhand der klinischen Fragestellung getroffen werden sollte. Während für einige Fragstellungen die Berechnung der resultierenden externen Gelenkmomente ausreichend ist, kann für andere Fragestellungen der Mehraufwand für die Berechnung von Gelenkkontaktkräften mithilfe der erweiterten muskuloskelettalen Modellen gerechtfertigt sein. Für die Beurteilung von dynamischen Gelenkbelastungen bei Patienteninnen und Patienten mit Valgusfehlstellung [7] sollte zum Beispiel die Berechnung der lateralen Kontaktkraft im Knie dem externen Gelenkmoment vorgezogen werden, da die externen Gelenkmomente nur eine geringe Korrelation mit den lateralen Kontaktkräften aufweisen.

Bei physiologischen Achsverhältnissen wirken $60-80 \%$ der gesamten intrinsischen Druckbelastung, die auf das Knie übertragen wird, auf das mediale Kompartiment $[1,7]$. In einer Studie an Patienten und Patientinnen mit Valgusfehlstellung konnte erstmals die Verteilung der Gelenkbelastung im medialen und lateralen Gelenkkompartiment mittels muskuloskelettaler Modellierung simuliert werden [7]. Diese Darstellung der Belastungsverteilung im medialen und lateralen
Gelenkkompartiment ist über die alleinige Bestimmung von Gelenkmomenten nicht möglich. Allerdings müssen bei der Berechnung von Kontaktkräften im Gelenk in der klinischen Routine viele Vereinfachungen und Modellannahmen getroffen werden, was eine gewisse Sachkenntnis voraussetzt.

\section{Messung der Muskelaktivität mittels Elektromyographie}

Die Elektromyographie widmet sich der Entstehung, Aufzeichnung und Analyse myoelektrischer Signale, die durch Zustandsvariationen der Muskelfasermembran generiert werden. Es wird also die willkürliche Muskelaktivierung während des Gehens mithilfe von auf der Haut angebrachten Oberflächenelektroden erfasst (• Abb. 1). Um eine quantitative EMG-Datenanalyse durchzuführen, muss ein spezifisches, statistisches Signalanalyseverfahren angewendet werden.

Bei männlichen Patienten mit Varusfehlstellung konnte mit dieser Messmethode der Nachweis erbracht werden, dass die Muskelaktivität des M. vastus medialis und lateralis in der Standphase beim Gehen im Vergleich zu einer gesunden Kontrollgruppe mit neutraler Beinachse höher ist [12]. Dies deutet darauf hin, dass Achsfehlstellungen des Knies zu einer veränderten muskulären Aktivität führen können. Die biomechanischen und klinischen Konsequenzen einer veränderten Muskelaktivität in Zusammenhang mit einer Achsfehlstellung sind noch nicht erforscht. Ebenso wenig ist bekannt, ob sich die muskuläre Situation nach einer operativen Korrektur der Achsfehlstellung normalisiert. Mit dieser Messmethodik eröffnen sich somit weitere Möglichkeiten, die Auswirkungen einer Achsabweichung auf die neuromuskuläre Aktivität und Ansteuerung zu untersuchen.

\section{Stellenwert der IGA bei der Beur- teilung von Achsfehlstellungen}

Die IGA ist eine international etablierte Methode zur detaillierten Befunderhebung der Gangfunktion. Sie liefert nicht nur wertvolle Zusatzinformationen zur konventionellen Diagnostik mit körperlicher Untersuchung und Bildgebung, sondern 


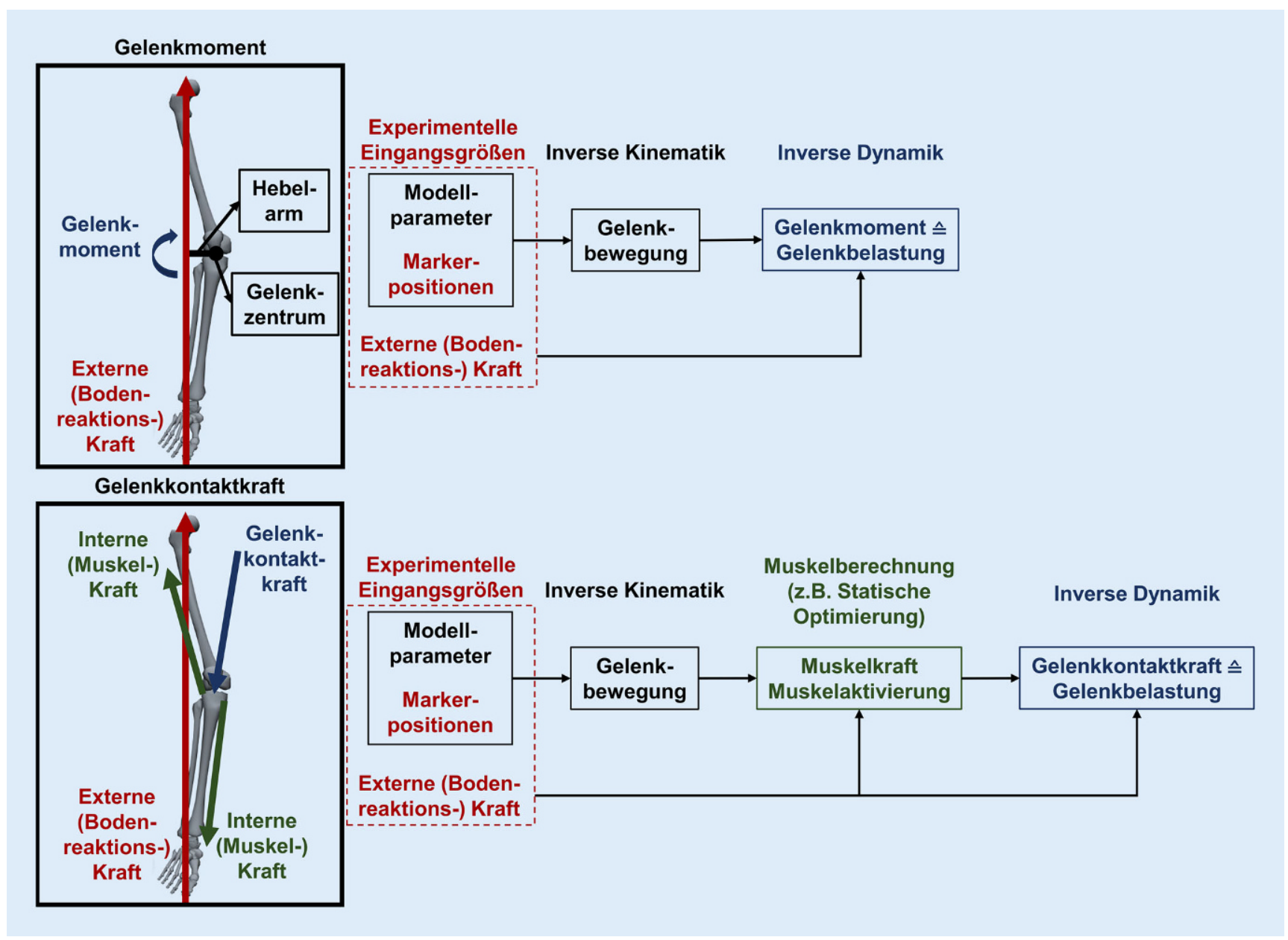

Abb. 3 ॥ Vereinfachte schematische Darstellung der Berechnung von Gelenkmomenten und Kontaktkräften im Gelenk. Zur Berechnung der Gelenkmomente werden äußere Kräfte, wie die Bodenreaktionskraft, und der zugehörige Hebelarm vom Gelenkzentrum zum Kraftvektor berücksichtigt. Im Gegensatz dazu werden bei der Berechnung der Kontaktkraft im Gelenk über die muskuloskelettale Modellierung nicht nur die äußeren Kräfte, sondern auch innere (Muskel-)Kräfte zur Berechnung der Gelenkbelastung berücksichtigt. Adaptiert nach [8]. Mit freundl. Genehmigung, @ 2020 Holder, Trinler, Meurer und Stief, alle Rechte vorbehalten

ist gerade im Bereich der Neuroorthopädie oft primär therapieentscheidend und prognostisch von hohem Wert $[5,28]$. Die Literaturlage belegt eindeutig, dass die IGA zum biomechanischen Verständnis von Gangpathologien und damit auch zur Behandlungsentscheidung und dem Operationserfolg beiträgt [10, 16, 29, 32].

Dagegen wird der IGA bei der Beurteilung von Achsfehlstellungen bisher wenig Bedeutung geschenkt. Dies liegt zum einen daran, dass nicht jede Klinik über eine entsprechendes Gang- oder Bewegungsanalyselabor verfügt. Zum anderen hat sich die statische Beurteilung von Achsfehlstellung anhand einer Ganzbein-Röntgenaufnahme im Stand etabliert und wird auch in der medizinischen Ausbildung weiterhin als Goldstandard angesehen. Die
Aufnahme dient der Bestimmung von Winkeln und Achsen anhand derer das Ausmaß der Fehlstellung bestimmt wird. Viele Kinder haben während ihres Wachstums physiologische Achsendeformitäten und die Frage, bis zu welchem Grad die Beinachse noch normal ist, ist teilweise schwierig zu beantworten. Gerade bei Grenzfällen bestehen Unsicherheiten, ab welchem Winkel bzw. welcher Achsabweichung die Beinachse als pathologisch anzusehen ist und wann operative Maßnahmen erforderlich sind. Hinzu kommt der mögliche Einfluss des Messfehlers bei der Durchführung bzw. Beurteilung der statischen Röntgenaufnahme. So kann das Ergebnis der Achsvermessung sowohl durch eine nicht korrekte Ausrichtung der Patella während der Röntgenaufnahme, als auch durch
Fehler bei der softwarebasierten Achsvermessung zum Teil beachtlich beeinflusst werden. Zudem lässt die rein statische Beurteilung nur begrenzt Rückschlüsse auf eine mögliche mechanische Überlastung des Kniegelenks bzw. eine spätere Arthroseentwicklung zu. Aufgrund dieser Kenntnisse liegt es auf der Hand, dass die statische Betrachtung der Achsfehlstellung durch eine dynamische Analyse ergänzt werden sollte.

Die dynamische Gelenkbelastung als Prädiktor für Arthroseentwicklung

Ein pathologisches frontales Kniegelenkmoment beim Gehen bei einer Varus- oder Valgusabweichung stellt einen ungünstigen Faktor hinsichtlich der Entwicklung 
degenerativer Gelenkveränderungen bis hin zur Entstehung von Arthrose im Kniegelenk dar [2, 9, 13-15, 22]. Daher sollte man bestrebt sein, diese dynamischen Belastungsverhältnisse auch zu messen und in die klinische Entscheidung zu integrieren. Die nichtinvasive Berechnung der dynamischen Belastungen im Kniegelenk ist mittels IGA möglich. Bei einer normalen Beinachse wirkt beim Gehen eine physiologische Varusbelastung. Das heißt, dass das mediale Gelenkkompartiment die Hauptlast trägt (ca. 60-80\% der gesamten intrinsischen Druckbelastung) $[1,7,20]$. Dies könnte eine Erklärung dafür sein, dass das mediale tibiofemorale Kompartiment häufiger von Arthrose betroffen ist als das laterale tibiofemorale Kompartiment [31].

Der Artikel von Andriacchi und Mündermann [2] gibt eine Übersicht zu Studien, die nachweisen konnten, dass das externe Knieadduktionsmoment (Varusmoment) während des Gehens ein Prädiktor für das Fortschreiten der Knorpeldegeneration im medialen Gelenkkompartiment bzw. die Entstehung von Arthrose im Kniegelenk ist. Dies bedeutet, dass das Arthroserisiko bei Gruppen mit erhöhten Knieadduktionsmomenten höher war als bei Kontrollgruppen mit physiologischen Knieadduktionsmomenten. Es konnte z.B. nachgewiesen werden, dass die Gelenkbelastung beim Gehen den Knorpelstoffwechsel bei gesunden Erwachsenen beeinflussen kann [14]. Diese Erkenntnisse bekräftigen die Beziehung zwischen der In-vivo-Belastung beim Gehen und der biologischen Reaktion des Knorpels. Weiterhin konnte in einer Studie bei Patienteninnen und Patienten mit Gonarthrose gezeigt werden, dass das Adduktionsmoment im Knie beim Gehen bei der Patientengruppe mit leichteren Arthrosezeichen signifikant niedriger war [15]. Weiterhin korreliert das Adduktionsmoment im Knie positiv mit dem Schweregrad (KellgrenLawrence-Einteilung) und negativ mit der Gelenkspaltbreite [22].

In einer prospektiven Studie von Miyazaki und Kollegen [13] wurde in einem 6-Jahres-Follow-Up die radiologische Verengung des Kniegelenkspaltes bei Patienten mit medialer Kniearthrose untersucht. Die Ergebnisse zeigen, dass das Fortschreiten der Arthrose von dem Ausgangswert des Knieadduktionsmomentes beim Ge- hen abhängig war. Mit anderen Worten, je größer das Knieadduktionsmoment - und damit die Belastung des medialen Kompartiments - beim Gehen, desto schneller schreitet die Arthrose im Kniegelenk voran. Die logistische Regressionsanalyse zeigt, dass das Risiko einer Progression der Kniearthrose bei einem Anstieg des Adduktionsmoments im Knie um 1\% um das 6,5fache anstieg.

Die dynamische Kniegelenkbelastung dient auch zur Vorhersage des Outcomes nach einer operativen Achskorrektur. Prodromos und Kollegen [19] konnten diesbezüglich zeigen, dass das präoperative Knieadduktionsmoment beim Gehen eine entscheidende Rolle für das langfristige Outcome (ca. 3 Jahre nach Operation) einer valgisierenden Umstellungsosteotomie spielt. In der Gruppe mit präoperativ erhöhten Adduktionsmomenten im Kniegelenk kam es zu einem Wiederauftreten der Varusfehlstellung (Rebound), während dies in der Gruppe mit signifikant niedrigeren präoperativen Knieadduktionsmomenten nicht der Fall war. Dabei spielte die statische Achsfehlstellung übrigens keine Rolle, da diese zum Zeitpunkt der Implantation in beiden Gruppen nicht unterschiedlich stark ausgeprägt war. Diese Studie zeigt sehr überzeugend, dass Informationen über die präoperativen Gelenkmomente beim Gehen wichtige Hinweise für das postoperative Outcome geben können. Sind die Adduktionsmomente im Knie bei einer Varusfehlstellung deutlich erhöht, müsste man aufgrund der Erkenntnisse folglich über eine geplante leichtgradige Überkorrektur der Beinachse (in Richtung Valgus) nachdenken, um das Risiko eines Rezidivs zu minimieren. Weitere Ausführungen zum optimalen Zeitpunkt der Metallentfernung sowie dem Rebound-Phänomen sind dem Beitrag „Häufigkeit und Prädiktoren für einen Rebound nach operativer Achskorrektur in der Frontalebene" in diesem Themenheft zu entnehmen.

\section{Diskrepanz zwischen statischer Achsfehlstellung und dynamischer Gelenkbelastung}

In einigen Studien konnte gezeigt werden, dass kein oder nur ein geringer Zusammenhang zwischen statischer Achs- vermessung und dynamischer Gelenkbelastung im Knie vorliegt [4, 6, 9]. Dies unterstreicht die Notwendigkeit einer dynamischen Bewertung der Belastungssituation im Kniegelenk. Individuelle Kompensationsmechanismen beim Gehen werden als Ursache für die mögliche Diskrepanz zwischen statischer Achsfehlstellung und dynamischer Kniegelenkbelastung angesehen [6, 7, 11, 24, 26]. Dazu gehören Veränderungen der unteren Extremitäten in der Transversalebene [6, 11, 24], wie z. B. eine vermehrte Außen- oder Innendrehung des Fußes [24, 30], oder auch eine Neigung des Rumpfes zur Standbeinseite beim Gehen [25]. Diese Kompensationsmechanismen können zu einer physiologischen Änderung des Kraftvektors in Relation zum Gelenkzentrums (Reduzierung des Hebelarms; - Abb. 3) führen und somit die Gelenkbelastung - trotz statischer Fehlstellung der Beinachse - reduzieren. Genauso kann bei einer statisch „normal aussehenden" Beinachse eine pathologische dynamische Gelenkbelastung vorliegen. Da solche Kompensationsmechanismen nicht in einem statischen Röntgenbild erfasst werden können, ist eine dynamische Beurteilung der Biomechanik der unteren Extremität notwendig. Die IGA kann somit für die klinische Entscheidungsfindung bei Achsfehlstellungen nützlich sein: Eine Wachstumslenkung durch Hemiepiphysiodesen im Kindesalter bzw. eine Umstellungsosteotomie im Erwachsenenalter sollte gerade bei Grenzfällen infrage gestellt werden, wenn die dynamische Belastungssituation im Kniegelenk trotz statischer Fehlstellung der Beinachse physiologisch ist (-Abb.4) Ansonsten kann es bei einer zuvor physiologischen Kniegelenkbelastung im ungünstigen Fall nach der operativen Achskorrektur zu einer pathologischen Kniegelenkbelastung kommen [4], was wiederum ein erhöhtes Risiko für das Fortschreiten der Knorpeldegeneration bzw. die Entstehung von Arthrose im Kniegelenk darstellt.

\section{Praxisbeispiel}

Die - Abb. 4a zeigt einen 14-jährigen Patient mit grenzwertiger, röntgenologischer Varusfehlstellung. Um die Frage nach der Notwendigkeit einer temporären Hemiepiphysiodese beurteilen zu 

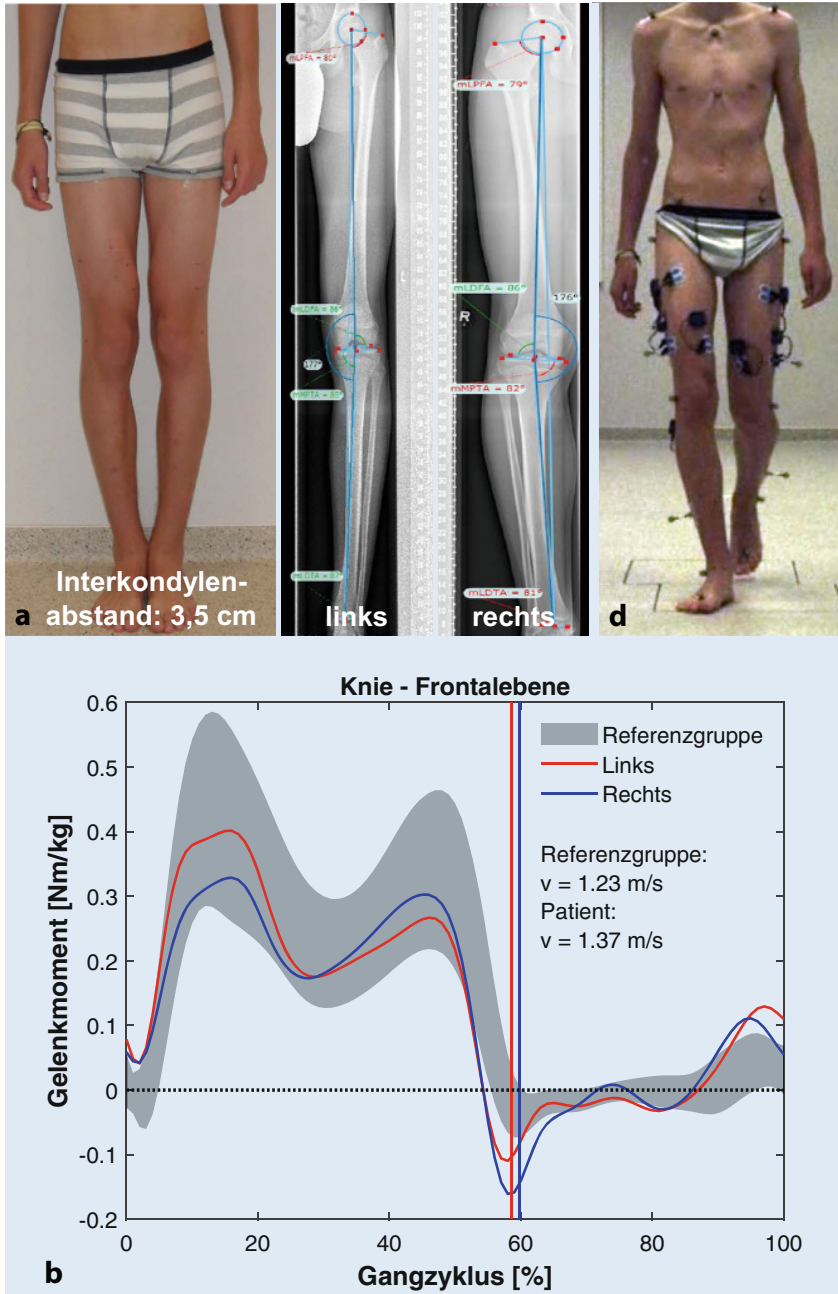

b

Fuß - Transversalebene

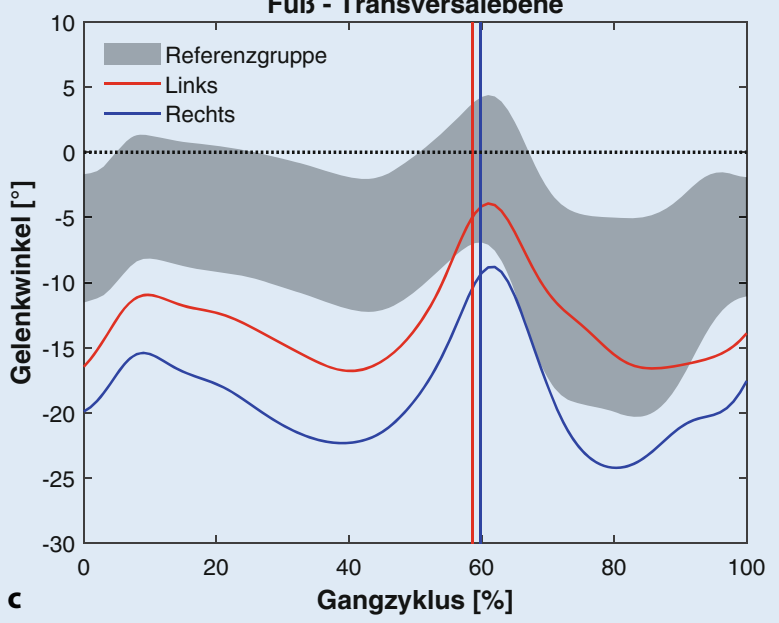

können, wurde eine IGA durchgeführt. Im Gegensatz zum röntgenologischen Befund zeigt die IGA im Vergleich zu einer gesunden Referenzgruppe physiologische Kniegelenkmomente in der Frontalebene (- Abb. 4b). Verantwortlich für die Diskrepanz zwischen statischer Achsfehlstellung und dynamischer Kniegelenkbelastung sind individuelle Kompensationsmechanismen beim Gehen. Im vorliegende Fallbeispiel ist dies eine vermehrte Außendrehung des Fußes im Vergleich zu einer gesunden Referenzgruppe (- Abb. 4c, d). Dies führt - trotz statischer Varusfehl-
Abb. 44 14-jähriger Patient mit grenzwertiger Varusfehlstellung und der Frage nach der Notwendigkeit einer temporären Hemiepiphysiodese. a Röntgenologische Beurteilung der Beinachse (mechanische Achsabweichung links: $16 \mathrm{~mm}$ medial, rechts: $19 \mathrm{~mm}$ medial; mechanischer femorotibialer Winkel links: $3^{\circ}$ Varus, rechts: $4^{\circ}$ Varus); $m L D F A_{\text {" }}$ mechanical lateral distal femoral angle", $m L P F A_{\text {,"mechanical lateral }}$ proximal femoral angle", $m M P T A$ "mechanical medial proximal tibial angle". b Dynamische Informationen aus der instrumentelle Ganganalyse: Im Gegensatz zum röntgenologischen Befund physiologische Kniegelenkmomente in der Frontalebene im Vergleich zu einer gesunden Referenzgruppe. Typische Kompensationsmechanismen beim Gehen sorgen dafür, dass die dynamische Gelenkbelastung beim Gehen nicht erhöht ist. Im vorliegende Fallbeispiel ist dies eine $\mathbf{c}$, d vermehrte Außendrehung des Fußes im Vergleich zu einer gesunden Referenzgruppe. Dies führt - trotz statischer Varusfehlstellung insbesondere in der 2. Hälfte der Standphase zu einer physiologischen Änderung des Kraftvektors in Relation zum Gelenkzentrums (Reduzierung des Hebelarms, - Abb. 3) und somit zu einer physiologischen Gelenkbelastung [30]

stellung - insbesondere in der 2. Hälfte der Standphase zu einer physiologischen Änderung des Kraftvektors in Relation zum Gelenkzentrum (Reduzierung des Hebelarms) und somit zu einer physiologischen Gelenkbelastung [30]. Aufgrund des grenzwertigen röntgenologischen Befundes und der physiologischen Belastungssituation im Kniegelenk durch die IGA besteht kein erhöhtes Risiko hinsichtlich der Entwicklung degenerativer Gelenkveränderungen bis hin zur Entstehung von Arthrose im Kniegelenk. Demzufolge besteht auch keine Indikation für eine temporäre Hemiepiphysiodese.

\section{Optimierung des Behandlungs- schemas bei der Beurteilung von Achsfehlstellungen durch Integration der IGA}

Obwohl die wissenschaftliche Evidenz der dynamischen Gelenkbelastung bei der Entstehung bzw. beim Fortschreiten von Arthrose im Kniegelenk gegeben ist, finden die oben ausgeführten Erkenntnisse bei der Beurteilung von Achsfehlstellungen im klinischen Alltag bisher kaum Anwendung. Der Zugang zu entsprechenden Ganganalysedaten hat sich in den letzten Jahren gerade im Bereich der Kinderorthopädie verbessert. Viele orthopädische Kliniken haben die Bedeutung 
Beurteilung der Beinachse in der Frontalebene unter Berücksichtigung der Statik (GanzbeinStandaufnahme im a.p.-Strahlengang) UND der Dynamik (Instrumentelle Ganganalyse)

1.

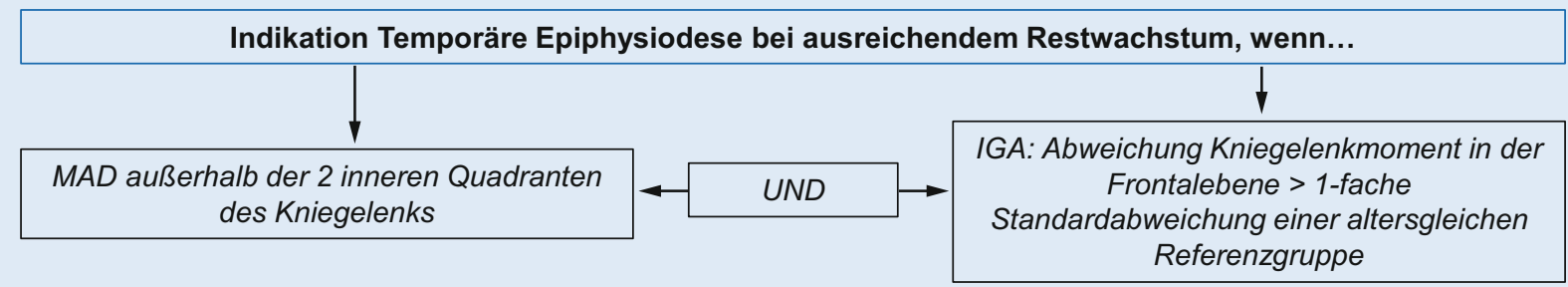

2.

\begin{tabular}{c}
$\begin{array}{c}\text { Regelmäßige Untersuchungen im Abstand von } 3 \text { Monaten zur Kontrolle der Beinachse und Festlegung des } \\
\text { optimalen Zeitpunktes der Metallentfernung }\end{array}$ \\
\hline Klinische Untersuchung / Interkondylen- bzw. Intermalleolenabstand sowie bei Bedarf... \\
\hline Röntgen
\end{tabular}

3.

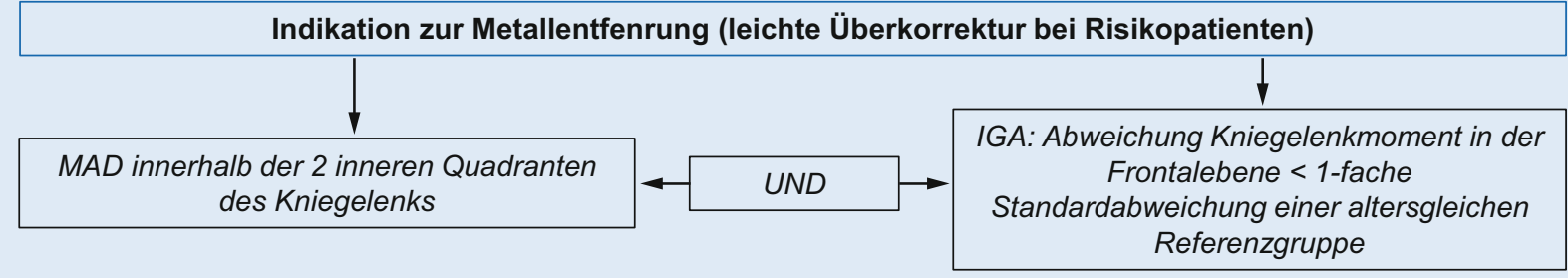

4.

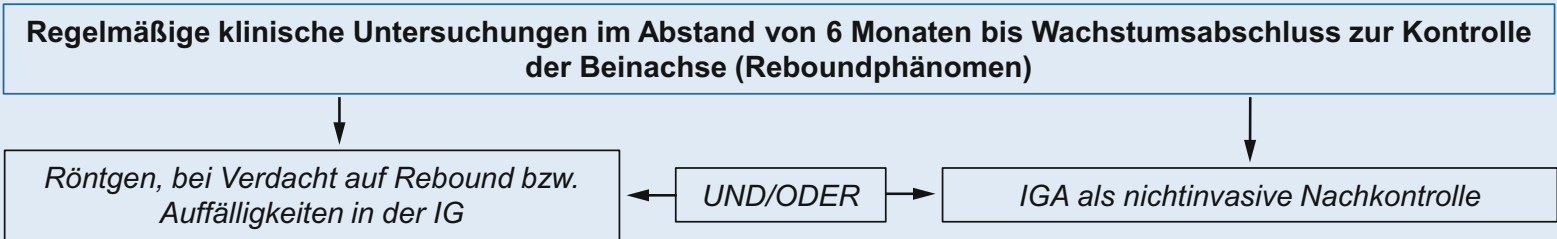

Abb. $5 \Delta$ Behandlungsalgorithmus bei grenzwertiger Achsfehlstellung (Genu varum und Genu valgum) zur exakteren Planung einer Wachstumslenkung im Kindesalter mittels temporären Hemiepiphysiodesen durch Integration der instrumentellen Ganganalyse (IGA). Beim Vergleich mit einer gesunden Referenzgruppe ist bei der IGA unbedingt zu empfehlen, dass die Erhebung der Referenzgruppe (bestehend aus mindestens 20 gesunden Probanden) im eigenen Labor erfolgt. Damit ein angemessener Vergleich mit den Patientendaten möglich ist, sollte die Referenzgruppe darüber hinaus im gleichen Alter sein und mit identischer Ganggeschwindigkeit gehen. Der Einfluss der Ganggeschwindigkeit auf kinematische und kinetische Gangparameter ist z. B. in der Studie von Schwartz und Kollegen [21] dargestellt. Mechanische Achsenabweichung (MAD) nach [3]

der dynamischen Betrachtungsweise erkannt und verfügen über die notwendige Messtechnik. Jetzt geht es in einem weiteren Schritt darum, die erhobenen Daten und Parameter in angemessener Weise zu interpretieren und die Erkenntnisse im Rahmen der klinischen Routine bei der Beurteilung von Achsfehlstellungen anzuwenden. Der Einsatz der IGA bedeutet keinesfalls einen Ersatz der konventionellen Röntgendiagnostik. Die IGA ist vielmehr als wertvolles Zusatzinstrument zu betrachten, das gerade bei grenzwertigen Befunden die Indikationsstellung für operative oder therapeutische Maßnahmen erleichtert und eine dynamische Beurteilung des Behandlungserfolges gestattet.

Erste, bisher nicht veröffentlichte Ergebnisse eines von der Deutsche Forschungsgemeinschaft geförderten Multicenter-Projekts mit Projektpartnern an der Klinik für Orthopädie (Friedrichsheim) des Universitätsklinikums Frankfurt am Main, an der Orthopädischen Kinderklinik in Aschau im Chiemgau, an der Orthopädischen Klinik der Medizinischen Hochschule in Hannover und am Waldkrankenhaus in Eisenberg/Universitätsklinikum Jena zeigen, dass die Methode der IGA zu einer Optimierung der Behandlung von kindlichen Achsfehlstellungen mittels temporärer Hemiepiphysiodesen führen und das Risiko für eine wiederkehrende Achsfehlstellung (Rebound-Phänomen, siehe Beitrag „Häufigkeit und Prädiktoren für einen Rebound nach operativer Achskorrektur in der Frontalebene" in diesem Themenheft) minimieren kann.

Ein weiteres Problem bei der temporären Hemiepiphysiodese ist die Notwendigkeit einer regelmäßigen, radiologischen 
Kontrolle der Entwicklung der Beinachse im Behandlungszeitraum. Dies ist notwendig, um den optimalen Zeitpunkt für die Implantatentfernung festzulegen und Überkorrekturen zu vermeiden. Somit sind die Patienteninnen und Patienten einer erhöhten Strahlenbelastung ausgesetzt. Möchte man in Zukunft mehr über das Rebound-Phänomen erfahren, sind zusätzliche regelmäßige Kontrollen nach Metallentfernung, im besten Fall bis Wachstumsabschluss, notwendig. Durch Anwendung der IGA ist es möglich, die statische Beinachse nichtinvasiv zu bestimmen. In einer kürzlich durchgeführten Studie [27] konnte der Nachweis erbracht werden, dass die statische Bestimmung der Beinachse mittels lichtreflektierender Marker über die IGA bei Patienten und Patientinnen mit Varus- oder Valgusfehlstellung sehr gut mit der radiologischen Achsfehlstellung (mechanischer femorotibialer Winkel) korreliert. Da auch die systematische Abweichung zwischen beiden Messmethoden gering ist, stellt die statische Messung über die IGA somit sowohl für den klinischen Alltag als auch für Forschungszwecke eine nichtinvasive Möglichkeit zur Beurteilung der frontalen Beinachse unter Belastung dar. Dies kann z. B. in Situationen nützlich und empfehlenswert sein, in denen die kumulative Strahlendosis während der Behandlungsperiode zu einem Problem wird, oder wenn aus ethischen Gründen keine röntgenologische Aufnahme durchgeführt werden kann (z. B. Vergleich der Beinachse mit einer gesunden Kontrollgruppe). Somit kann die IGA auch als "VorabScreening-Tool“ zur Identifizierung von Patienteninnen und Patienten mit auffälliger Beinachse bzw. pathologischer Gelenkbelastung dienen, was dann im Bedarfsfall mittels Röntgenbild weiter abgeklärt werden kann.

\section{I) Durch Anwendung der IGA ist es möglich, die statische Beinachse nichtinvasiv zu bestimmen}

Um eine umfassende Beurteilung einer Achsfehlstellung zu erhalten, sollte in Zukunft der Austausch zwischen biomechanischer Forschung und klinischer Anwendung intensiviert und ein einheitliches Konzept zur Optimierung der Behandlung von kindlichen Achsfehlstellungen unter Berücksichtigung von Statik (Röntgen) und Dynamik (IGA) etabliert werden. Durch Hinzunahme der IGA lässt sich insbesondere bei Grenzfällen die Therapieplanung bzw. Operationsindikation bekräftigen oder optimieren. Selbstverständlich müssen zusätzlich klinische Beschwerden, wie Mobilitätseinschränkungen und Schmerzen, in die Indikationsprüfung einbezogen werden. Steht im Kindesalter aufgrund einer Achsfehlstellung eine kniegelenksnahe Wachstumslenkung durch eine temporäre Hemiepiphysiodese im Raum, schlagen die Autoren vor allem für Patienten und Patienteninnen mit grenzwertiger Achsfehlstellung den in - Abb. 5 dargestellten Behandlungsalgorithmus vor.

Wie dem Behandlungsalgorithmus zu entnehmen ist, können dynamische Messungen auch hilfreich sein, wenn es darum geht, den optimalen Zeitpunkt für die Entfernung der Platten bei temporären Hemiepiphysiodesen zu bestimmen. Die IGA kann dabei, neben der statischen Achsvermessung, zur Absicherung dienen, ob die dynamische Belastungssituation im Kniegelenk normalisiert wurde. Bei einer Diskrepanz zwischen statischer Beinachse und dynamischer Kniegelenkbelastung kann die IGA potenzielle Kompensationsmechanismen beim Gehen aufdecken und somit Erklärungen für diese Diskrepanz liefern. Außerdem können bei ungünstigen Belastungssituationen Empfehlungen für weitere Behandlungsansätze, wie z.B. die Versorgung mit entsprechenden Einlagen bei Valgusfehlstellung der Beinachse in Kombination mit einem Knick-SenkFuß, abgeleitet werden und so eine natürliche Belastungssituation im Kniegelenk erreicht werden.

\section{Fazit für die Praxis}

- Die Ganzbein-Röntgenaufnahme im Stand lässt nur bedingt Rückschlüsse auf eine mögliche mechanische Überlastung des Kniegelenks bzw. eine spätere Arthroseentwicklung zu.

- Die instrumentelle Ganganalyse (IGA) liefert über die Berechnung der dynamischen Gelenkbelastungen wichtige Erkenntnisse im Hinblick auf das Fortschreiten der Knorpeldegeneration bzw. die Entstehung von Arthrose im Kniegelenk und ergänzt somit die statische Beurteilung der Beinachse.
- Individuelle Kompensationsmechanismen beim Gehen können eine mögliche Diskrepanz zwischen statischer Achsfehlstellung und dynamischer Kniegelenkbelastung erklären.

- Zur Optimierung der Behandlung von kindlichen Achsfehlstellungen sollte in Zukunft ein einheitliches Konzept unter Berücksichtigung von Statik (Röntgen) und Dynamik (IGA) etabliert werden. Dieser Beitrag liefert einen Vorschlag für einen entsprechenden Behandlungsalgorithmus für kniegelenksnahe Wachstumslenkungen mittels temporärer Hemiepiphysiodese.

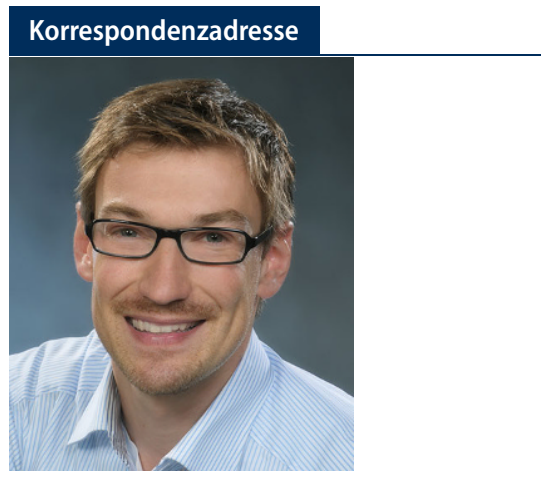

\section{PD. Dr. habil. Felix Stief}

Klinik für Orthopädie (Friedrichsheim), Universitätsklinikum Frankfurt Marienburgstraße 2, 60528 Frankfurt am Main, Deutschland

felix.stief@kgu.de

Danksagung. Dieser Beitrag ist im Rahmen eines von der Deutschen Forschungsgemeinschaft (DFG) geförderten Projekts entstanden (Projektnummer: 403837822)

\section{Einhaltung ethischer Richtlinien}

Interessenkonflikt. F. Stief, J. Holder, H. Böhm und A. Meurer geben an, dass kein Interessenkonflikt besteht.

Für diesen Beitrag wurden von den Autoren keine Studien an Menschen oder Tieren durchgeführt. Für die aufgeführten Studien gelten die jeweils dort angegebenen ethischen Richtlinien.

Open Access. Dieser Artikel wird unter der Creative Commons Namensnennung 4.0 International Lizenz veröffentlicht, welche die Nutzung, Vervielfältigung Bearbeitung, Verbreitung und Wiedergabe in jeglichem Medium und Format erlaubt, sofern Sie den/die ursprünglichen Autor(en) und die Quelle ordnungsgemäß nennen, einen Link zur Creative Commons Lizenz beifügen und angeben, ob Änderungen vorgenommen wurden.

Die in diesem Artikel enthaltenen Bilder und sonstiges Drittmaterial unterliegen ebenfalls der genannten 
Creative Commons Lizenz, sofern sich aus der Abbildungslegende nichts anderes ergibt. Sofern das betreffende Material nicht unter der genannten Creative Commons Lizenz steht und die betreffende Handlung nicht nach gesetzlichen Vorschriften erlaubt ist, ist für die oben aufgeführten Weiterverwendungen des $\mathrm{Ma}$ terials die Einwilligung des jeweiligen Rechteinhabers einzuholen.

Weitere Details zur Lizenz entnehmen Sie bitte der Lizenzinformation auf http://creativecommons.org/ licenses/by/4.0/deed.de.

\section{Literatur}

1. Andriacchi TP (1994) Dynamics of knee malalignment. Orthop Clin North Am 25:395-403

2. Andriacchi TP, Mündermann A (2006) The role of ambulatory mechanics in the initiation and progression of knee osteoarthritis. Curr Opin Rheumatol 18:514-518

3. Ballal MS, Bruce CE, Nayagam S (2010) Correcting genu varum and genu valgum in children by guided growth: temporary hemiepiphysiodesis using tension band plates. J Bone Joint Surg Br 92:273-276

4. Böhm H, StiefF, Sander Ket al (2015) Correction of static axial alignment in children with kneevarus or valgus deformities through guided growth: Does it also correct dynamic frontal plane moments during walking? Gait Posture 42:394-397

5. Dreher T, Wolf SI, Heitzmann D et al (2012) Longterm outcome of femoral derotation osteotomy in children with spastic diplegia. Gait Posture 36:467-470

6. Farr S, Kranzl A, PablikE et al (2014) Functional and radiographic consideration of lower limb malalignment in children and adolescents with idiopathic genu valgum. J Orthop Res 32:1362-1370

7. Holder J, Feja Z, Van Drongelen S et al (2020) Effect of guided growth intervention on static leg alignment and dynamic knee contact forces during gait. Gait Posture 78:80-88

8. Holder J, Trinler U, Meurer A et al (2020) A Systematic Review of the Associations Between Inverse Dynamics and Musculoskeletal Modeling to Investigate Joint Loading in a Clinical Environment. Front Bioeng Biotechnol 8:603907

9. Hurwitz DE, Ryals AB, Case JP et al (2002) The knee adduction moment during gait in subjects with knee osteoarthritis is more closely correlated with static alignment than radiographic disease severity, toe out angle and pain. J Orthop Res 20:101-107

10. Kay RM, Dennis $S$, Rethlefsen $S$ et al (2000) The effect of preoperative gait analysis on orthopaedic decision making. Clin Orthop Relat Res 37(2):217-222

11. MacWilliams BA, McMulkin ML, Baird GO et al (2010) Distal tibial rotation osteotomies normalize frontal plane knee moments.J Bone Joint Surg Am 92:2835-2842

12. Madadi-Shad $M$, Jafarnezhadgero $A$, Zago $M$ et al (2019) Effects of varus knee alignment on gait biomechanics and lower limb muscle activity in boys: A cross sectional study. Gait Posture 72:69-75

13. Miyazaki T, Wada M, Kawahara $\mathrm{H}$ et al (2002) Dynamic load at baseline can predict radiographic disease progression in medial compartment knee osteoarthritis. Ann Rheum Dis 61:617-622

14. Mündermann A, Dyrby CO, Andriacchi TP et al (2005) Serum concentration of cartilage oli-

\section{Dynamic analysis of joint loading due to leg axis deformity in the frontal plane. Relevance of instrumented gait analysis}

Background: Instrumented 3D gait analysis (IGA) has been established for the functional evaluation of orthopedic diseases. It can provide valuable additional information beyond conventional static radiographic diagnostics and, thus, contributes to treatment decisions and a successful surgical outcome. Regarding the assessment of leg axis deformities IGA is currently only used in a few specialized centers.

Practice: This article describes the methods used by IGA and shows its benefit for the treatment of leg axis deformities of the knee in the frontal plane. In particular, the calculation of dynamic joint loads provides important insights regarding the development of degenerative joint deformities in the knee joint and, thus, complements the static assessment of the leg axis. A new treatment algorithm for guided growth intervention in children and adolescents by temporary epiphysiodesis is presented. IGA can be particularly useful for clinical decision-making in borderline cases. If there is a discrepancy between the static leg axis and dynamic knee joint loading, IGA can reveal potential compensatory mechanisms during walking.

\section{Keywords}

Leg malalignment - Clinical decision-making · Genu valgum · Genu varum · Knee

gomeric matrix protein (COMP) is sensitive to physiological cyclic loading in healthy adults. Osteoarthritis Cartilage 13:34-38

15. Mündermann A, Dyrby CO, Hurwitz DE et al (2004) Potential strategies to reduce medial compartment loading in patients with knee osteoarthritis of varying severity: reduced walking speed. Arthritis Rheum 50:1172-1178

16. Nardone A, Svehlik M, Rhodes J et al (2020) Perspective on the Clinical Impact of Instrumented Motion Analysis by the Gait and Posture Affiliate Society Presidents. Gait Posture 82:106-107

17. Oppelt K, Hogan A, Stief F et al (2020) Movement Analysis in Orthopedics and Trauma Surgery Measurement Systems and Clinical Applications. ZOrthop Unfall 158:304-317

18. Perry J, Burnfield JM (2010) Gait Analysis. Normal and pathological function, 2. Aufl. SLACK Incorporated, Thorofare

19. Prodromos CC, Andriacchi TP, Galante JO (1985) A relationship between gait and clinical changes following high tibial osteotomy. J Bone Joint Surg Am 67:1188-1194

20. Schipplein OD, Andriacchi TP (1991) Interaction between active and passive knee stabilizers during level walking. J Orthop Res 9:113-119

21. Schwartz MH, Rozumalski A, Trost JP (2008) The effect of walking speed on the gait of typically developing children. J Biomech 41:1639-1650

22. Sharma L, Hurwitz DE, Thonar EJ et al (1998) Knee adduction moment, serum hyaluronan level, and disease severity in medial tibiofemoral osteoarthritis. Arthritis Rheum 41:1233-1240

23. Stief $F$ (2018) Variations of Marker Sets and Models for Standard Gait Analysis. In:Müller B, WolfS (Hrsg) Handbook of Human Motion. Springer, Cham, S509-526

24. Stief F, Böhm H, Dussa CU et al (2014) Effect of lower limb malalignment in the frontal plane on transverse plane mechanics during gait in young individuals with varus knee alignment. Knee 21:688-693

25. Stief F, Böhm H, Ebert C et al (2014) Effect of compensatory trunk movements on knee and hip joint loading during gait in children with different orthopedic pathologies. Gait Posture 39:859-864
26. Stief F, Böhm H, Schwirtz A et al (2011) Dynamic loading of the knee and hip joint and compensatory strategies in children and adolescents with varus malalignment. Gait Posture 33:490-495

27. Stief $F$, Feja Z, Holder J et al (2020) Noninvasive determination of frontal plane lower limb alignment using motion capture technique - An alternative for full-length radiographs in young patients treated by a temporary hemiepiphysiodesis? Gait Posture 79:26-32

28. Svehlík M, Kraus T, Steinwender G et al (2012) The Baumann procedure to correct equinus gait in children with diplegic cerebral palsy: long-term results. J Bone Joint Surg Br 94:1143-1147

29. Theologis T, Wright J (2015) Is 3. D Gait Analysis Essential? By Profr James Wright: Introd By Mr Tim Theol Gait Posture 42:227-229

30. Wang S, Mo S, Chung RCK et al (im Druck) How foot progression angle affects knee adduction moment and angular impulse in people with and without medial knee osteoarthritis: a metaanalysis. Arthritis Care Res. Im Druck. https://doi. org/10.1002/acr.24420

31. Wise BL, Niu J, Yang M et al (2012) Patterns of compartment involvement in tibiofemoral osteoarthritis in men and women and in whites and African Americans. Arthritis Care Res (Hoboken) 64:847-852

32. Wren TA, Otsuka NY, Bowen RE et al (2011) Influence of gait analysis on decision-making for lower extremity orthopaedic surgery: Baseline data from a randomized controlled trial. Gait Posture 34:364-369 